\title{
UCSF
}

\section{Postprints from the CTCRE}

\section{Title}

Association of the California tobacco control program with declines in lung cancer incidence

Permalink

https://escholarship.org/uc/item/38f90581

Journal

Cancer Causes \& Control, 15(7)

ISSN

0957-5243

\section{Authors}

Barnoya, J

Glantz, Stanton A., Ph.D.

Publication Date

2004-09-01

Peer reviewed 


\title{
Association of the California tobacco control program with declines in lung cancer incidence ${ }^{\star}$
}

\author{
Joaquin Barnoya \& Stanton Glantz* \\ Center for Tobacco Control Research and Education, Cardiovascular Research Institute, Institute for Health Policy \\ Studies, University of California, San Francisco
}

Received 17 October 2003; accepted in revised form 5 April 2004

Key words: lung cancer, epidemiology, tobacco control.

\begin{abstract}
Objective: The California tobacco control program enacted in 1988 has been associated with declines in smoking and heart disease mortality. Since smoking also causes lung cancer, we investigated whether the program was associated with a decline in lung and other cancer incidence.

Methods: Age-adjusted incidence rates of lung and bladder cancer (which are caused by smoking) and prostate and brain cancer (which are not) in the San Francisco-Oakland (SFO) Surveillance Epidemiology End Results (SEER) registry and other eight SEER registries from 1975 to 1999 were fitted in multiple regression analyses accounting for the time lag between program implementation and its effects on cancer incidence. Cigarette consumption over time was also analyzed and related to lung cancer incidence.

Results: With a one year lag, the incidence of lung cancer in SFO, relative to eight other SEER registries, fell significantly below that predicted from the pre-1990 rates, by -0.981 (cases $/ 100,000 /$ year)/year $(p=0.001)$. With a three year lag, the incidence of bladder cancer fell by -0.234 (cases/100,000/year)/year $(p=0.066)$. No association of the program was observed on prostate or brain cancers in SFO. During the first decade, the Program was associated with about a $6 \%$ reduction in lung cancer incidence; state-wide that corresponds to about 11,000 cases avoided. Conclusion: A comprehensive tobacco control program is associated with a lower incidence of lung cancer.
\end{abstract}

\section{Introduction}

In 1988, California enacted a comprehensive tobacco control program [1] that attacked the tobacco industry and stressed clean indoor air and policies to create smoke-free environments [2]. The program accelerated the decline in both smoking prevalence $[3,4]$ and per capita cigarette consumption $[5,6]$ compared with the rest of the United States. The decline in cigarette consumption was associated with a similar drop in heart disease death rates [5, 6], consistent with the fact that the risk of heart disease death falls rapidly after smoking cessation [4].

* This work was supported by the American Legacy Foundation and National Cancer Institute Grant CA-61021.

* Address correspondence to: Stanton A. Glantz, PhD, Professor of Medicine, Box 1390, University of California, San Francisco, CA 94143, USA. Ph.: 415-476-3893; Fax: 415-514-9345; E-mail: glantz@) medicine.ucsf.edu
Lung cancer is the second most diagnosed cancer and the leading cause of cancer death in the United States [7]. In the United States, the incidence rate began to decline for men after 1990 and leveled off for women in 1997 [7-9]. With the vast majority of lung cancer caused by smoking, one would expect to see a similar association of the tobacco control program on lung cancer as was observed for heart disease [5,6], allowing for an appropriate lag between changes in cigarette consumption and cancer incidence.

Consistent with this expectation, a faster drop in lung cancer incidence in California has been documented. Jemal et al. showed that lung cancer death rates in young adults (age 30-39 years) fell in states with high tobacco control efforts at a time that they were increasing in states with low tobacco control efforts during the 1990's [10]. They reported that California had an $18 \%$ decrease in the lung cancer rates from the 1990-1994 to the 1995-1999 period [10]. 
The Centers for Disease Control and Prevention (CDC) reported that lung cancer rates in California fell faster than the rest of the United States during the period 1988-1997 [11]. In addition, according to the California Department of Health Services, lung cancer incidence rates decreased $19.5 \%$ in the $1988-1999$ period; in 1999 the rates in California were 10.4\% lower than the national incidence rate [12]. The confidence with which these reports could attribute the changes in lung cancer rates to the tobacco control program was limited, however, because there were no statewide data available prior to 1988 when the California Tobacco Control Program started to establish baseline differences or pre-existing trends in cancer incidence. We sought to do a comparison that took into account long-term trends in cancer incidence that predated creation of the tobacco control program.

There are data on cancer incidence available since 1975 from the Surveillance, Epidemiology, and End Results (SEER) database (http://seer.cancer.gov) for nine geographical areas, including the San FranciscoOakland (SFO) area in California. Using the same analytical approach as the earlier study of the relationship between the California Tobacco Control Program and heart disease [5, 6], we used the SEER data to test if the Program was associated with the decline in lung cancer incidence. This same model was used for bladder cancer, another smoking-caused cancer [8, 12, 13], and prostate $[14,15]$ and brain and other nervous tissue cancer [16], which are not caused by smoking. These results substantially strengthen the earlier conclusions $[10,11]$ that tobacco control programs not only reduce smoking, but have a substantial effect on lung, and, perhaps, other cancer incidence.

\section{Methods}

Data

We obtained crude and age-adjusted incidence rates for lung (and bronchus) cancer, bladder cancer, prostate cancer, and brain and other nervous tissue cancer from the SEER database for the nine geographical areas with data since 1975: the states of Connecticut, Iowa, New Mexico, Utah, and Hawaii; the metropolitan areas of Detroit, Michigan, and SFO, California. SFO includes Alameda, Contra Costa, Marin, San Francisco, and San Mateo counties [17]. None of the eight SEER geographical areas outside SFO had tobacco control programs. Using age-adjusted lung cancer incidence controls for changes in the age distribution of the population over time as well as population growth.
To estimate the association of the program on tobacco consumption we selected per capita cigarette sales [18]. We obtained population data for the US from the National Center for Health Statistics [19] and for California from the California Department of Health Services [20], and for SFO from SEER [17].

\section{Statistical Analysis}

We modeled the association of the Program as a linear increase in effect over time after the Program was enacted in 1988 allowing for a time lag before changes in cancer rates are manifest. Rather than simply doing a traditional time series analysis of the SFO data, we used the lung cancer incidence rates (as a function of time) in the non-SFO areas as independent variables in the regression analysis. (This analysis is precisely the same approach used in the earlier analysis of the association between the California Tobacco Control Program and heart disease deaths in California [5, 6].) Using ageadjusted lung cancer incidence outside California as independent variables in the analysis provides a better control for underlying secular trends in cancer incidence, changes in smoking and other risk factors in the US population as a whole, changes in rates of diagnosis, and other unknown factors. We do not treat cigarette consumption as one of the explicit independent variables in the regression analysis to predict trends in lung cancer incidence. Rather, the differences in cigarette consumption patterns between SFO and the other SEER registries are implicitly accounted for by using lung cancer incidence rates in the other SEER registries as independent variables in the regression equation for SFO. Doing this (as opposed to including cigarette consumption directly) also implicitly integrates the effects of the lag between changes in smoking behavior and lung cancer risk in the model.

Similar to the earlier analysis of heart disease [5], we used the regression equation:

$$
\begin{aligned}
I_{\mathrm{SFO}}(t)= & b_{0}+b_{\text {Connecticut }} I_{\text {Connecticut }}(t)+b_{\text {Detroit }} I_{\text {Detroit }}(t) \\
& +b_{\text {Hawaii }} I_{\text {Hawaii }}(t)+b_{\text {Iowa }} I_{\text {Iowa }}(t) \\
& +b_{\text {NewMexico }} I_{\text {NewMexico }}(t)+b_{\text {Seattle }} I_{\text {Seattle }}(t) \\
& +b_{\text {Utah }} I_{\text {Utah }}(t)+b_{\text {Atlanta }} I_{\text {Atlanta }}(t) \\
& +b_{\mathrm{TCP}} L_{\mathrm{k}}(t)+\epsilon
\end{aligned}
$$

where $I_{i}(t)$ is the age-adjusted cancer incidence rate in year $\mathrm{t}$ at location $i$, and $L_{k}(t)=\max (t-1988-k, 0)$ where $k$ is the lag (in years) between when the Program was enacted in 1988 and when there was a discernable acceleration in the decline of cancer incidence from what 
would be predicted based on patterns established before the Program. $\epsilon$ is the random error term, assumed to be drawn from a stationary normal distribution. A significant value of the coefficient associated with the Tobacco Control Program $\left(b_{\mathrm{TCP}}\right)$ would indicate that there is a difference that grows linearly with time in cancer incidence in SFO after the California Tobacco Control Program started. (This analysis differs slightly from the earlier analysis of heart disease [5] because it accounts for the lag effect that is seen with cancer, but not with heart disease.)

If there is no lag, $k=0$, and the cancer rates would start to change as soon as the Tobacco Control Program began. To select the appropriate lag, we conducted a stepwise regression to predict lung cancer incidence in SFO in which we forced the lung cancer incidence rates for the eight other SEER registries into the model, then allowed the algorithm to include a lagged effect for the Program with lags ranging from -1 to +5 (i.e. putting the effective $t=0$ point for Program effect at 1987-1993).

For lung cancer, to test whether it is reasonable to associate a significant value of $b_{\mathrm{TCP}}$ with the California Tobacco Control Program, we modeled the incidence rate for the other 8 SEER registries using the same equation, excluding SFO. Our hypothesis was that if we would include the Program in the regression equation of the other registries, $b_{\mathrm{TCP}}$ would not be statistically significant. For example, for Connecticut,

$$
\begin{aligned}
I_{\text {Connecticut }}(t)= & b_{0}+b_{\text {Detroit }} I_{\text {Detroit }}(t)+b_{\text {Hawaii }} I_{\text {Hawaii }}(t) \\
& +b_{\text {Iowa }} I_{\text {Iowa }}(t)+b_{\text {NewMexico }} I_{\text {NewMexico }}(t) \\
& +b_{\text {Seattle }} I_{\text {Seattle }}(t)+b_{\mathrm{Utah}} I_{\mathrm{Utah}}(t) \\
& +b_{\text {Atlanta }} I_{\text {Atlanta }}(t)+b_{\mathrm{TCP}} L_{\mathrm{k}}(t)+\epsilon
\end{aligned}
$$

All comparisons were done with the same lag $\mathrm{k}$ identified from the SFO data.

Given that we did $m=9$ comparisons for lung cancer, we adjusted the $P$-values for the family of 8 comparisons with $1-(1-\alpha)^{\mathrm{m}}$ where $\alpha=p$ associated with $b_{\mathrm{TCP}}$ and $m=9$. All p-values are double-sided.

We did separate analysis by gender to account for possible gender differences in lung cancer incidence. In addition, during the period of time the Program was in effect there was a substantial increase in California's Hispanic population, which has lower smoking and lung cancer rates than non-Hispanic whites [21]. To investigate whether the changes in lung cancer incidence we observed were an artifact due to the increase in Hispanic immigration, we conducted a separate analysis for nonHispanic whites only.

We estimated the cases of cancer prevented by the Tobacco Control Program by calculating the difference in the age-adjusted incidence in SFO and the predicted rate in the absence of the Program obtained by setting $b_{\mathrm{TCP}}$ to 0 . For each year we multiplied this difference by the ratio of the crude rate to the age-adjusted cancer incidence rate for SFO to estimate the number of cases prevented for that year, then summed these crude differences over time. We calculated the percentage of cases prevented by the Program each year as $100 \%$ minus the ratio of the actual age-adjusted incidence by the predicted rate if the Program had not been instituted.

We did a similar analysis for the relationship between the Program and the incidence of bladder, prostate, and brain cancer for SFO only. Since we only did one test for each cancer site, we did not adjust the $P$ values associated with $b_{\mathrm{TCP}}$. The lag $\mathrm{k}$ was optimized for each cancer site separately.

In addition, we report changes in cigarette consumption in California associated with the Program. Similar to the earlier analysis [5], we used the per capita cigarette consumption in the rest of the United States (excluding California), together with $b_{\mathrm{TCP}} L_{0}(t)$ to model the effects of the Program.

\section{Results}

\section{Lung cancer}

The best model fit for lung cancer incidence in SFO was the one with a $k=+1$ year lag. Our model provided an excellent fit for age-adjusted lung cancer incidence in all SEER registries, with correlation coefficients between 0.77 and 0.98 (Table 1 and Figure 1) and no significant evidence of serial correlation in the residuals (Durbin-

\begin{tabular}{|c|c|c|c|c|}
\hline \multirow[t]{2}{*}{$\begin{array}{l}\text { SEER } \\
\text { Registry }\end{array}$} & $b_{\mathrm{TCP}}{ }^{\mathrm{b}}$ & $\begin{array}{l}\text { Standard } \\
\text { error }\end{array}$ & $\begin{array}{l}\text { Adjusted } p \\
\text { value for } b_{\mathrm{TCP}}\end{array}$ & \multirow[t]{2}{*}{$r$} \\
\hline & \multicolumn{2}{|c|}{$($ cases $/ 100,000 / \mathrm{yr}) / \mathrm{yr}$} & & \\
\hline $\mathrm{SFO}$ & -0.981 & 0.122 & 0.001 & 0.96 \\
\hline Connecticut & 0.148 & 0.154 & 0.979 & 0.98 \\
\hline Detroit & -0.485 & 0.204 & 0.237 & 0.97 \\
\hline Hawaii & 0.019 & 0.274 & 0.999 & 0.77 \\
\hline Iowa & 0.227 & 0.189 & 0.924 & 0.98 \\
\hline New Mexico & -0.230 & 0.172 & 0.868 & 0.86 \\
\hline Seattle & 0.201 & 0.193 & 0.965 & 0.96 \\
\hline Utah & 0.053 & 0.127 & 0.999 & 0.81 \\
\hline Atlanta & -0.061 & 0.253 & 0.999 & 0.96 \\
\hline
\end{tabular}
Watson statistics between 1.5 and 2.6).

Table 1. Regression results for age-adjusted incidence rate of lung cancer in $\mathrm{SFO}^{\mathrm{a}}$

\footnotetext{
a SFO: San Francisco/Oakland.

${ }^{\mathrm{b}} b_{\mathrm{TCP}}$ : Coefficient associated with the Tobacco Control Program in the model for each one of the SEER registries.
} 


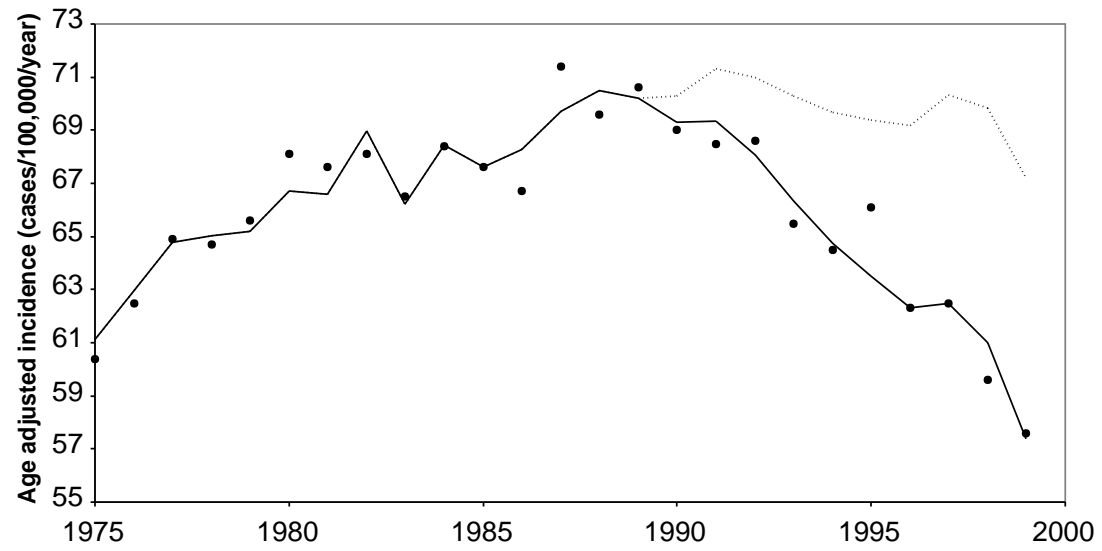

Fig. 1. Age-adjusted incidence (dots) for lung cancer in SFO predicted from the regression model (see text) with (solid line) and without (dotted line) the Tobacco Control Program.

The California Tobacco Control Program was associated with a significant decrease in the age-adjusted incidence rate for lung cancer. $b_{\mathrm{TCP}}=-0.981$ (cases/ $100,000 /$ year $) /$ year $(p=0.001)$, indicating that after the introduction of the Program, there was a significantly greater rate of decline in the age-adjusted lung cancer incidence rate in SFO beyond what would be predicted from the historical relationship between the incidence in SFO and the other eight SEER registries before the Program was implemented (Table 1 and Figure 1). $b_{\mathrm{TCP}}$ was not significant in any of the other eight SEER registries (Table 1) where there was no tobacco control program. This result supports the conclusion that the significant result in SFO is due to an association of the Program with lung cancer rates and not a statistical artifact.

For females, $b_{\mathrm{TCP}}=-0.775($ cases $/ 100,000 /$ year $) /$ year $(p=0.001)$ and for males $b_{\mathrm{TCP}}=-2.836($ cases $/ 100,000 /$ year)/year $(p=0.001)$, indicating that the program was associated with the decrease in age-adjusted lung cancer incidence in both genders. Regarding non-Hispanic whites, the magnitude of the Program association was similar to that observed in the entire population: $b_{\mathrm{TCP}}$ $=-0.766($ cases $/ 100,000 /$ year $) /$ year $(p=0.001)$. Thus, it does not appear that the result we found was due to the increase in the Hispanic population.

\section{Bladder cancer}

For bladder cancer, the best model was the one with a $k=+3$ year lag. $b_{\mathrm{TCP}}=-0.235($ cases $/ 100,000 /$ year $) /$ year, which approached statistical significance $(p=0.066)$.

\section{Non-tobacco cancers}

There was no significant association between the California Tobacco Control Program on the age-adjusted incidence of prostate ( $p=0.334$ for $b_{\mathrm{TCP}}$ with a two year lag) or brain ( $p=0.078$ for $b_{\text {TCP }}$ with a five year lag) cancers, which are not caused by tobacco.

\section{Cigarette consumption}

The percentage reductions in lung cancer over time tracked the reduction in cigarette consumption, with some lag and with the effects accumulating over time (Figure 2). The regression equation for per capita cigarette consumption in California was $\mathrm{CA}=-54.5$ $\mathrm{pack} / \mathrm{yr}+1.23(\mathrm{US}-\mathrm{CA})-0.794 L_{0}(t)$, where $\mathrm{CA}=$ California per capita consumption and $($ US-CA) $=$ per capita consumption in the rest of the US.

\section{Lung cancer cases avoided}

The total number of cases avoided for lung cancer in SFO over the ten year period from 1989 to 1999 was 2036 , about $6 \%$ of the total number of cases; in the last year of our analysis (1999) we estimate that approximately $14 \%$ of lung cancer cases were avoided (Figure 2).

Even though there may be some demographic differences between SFO and the rest of the state, the changes in cancer incidence are closely related to state-wide patterns. After 1988, when statewide data are available, there was good correlation between incidence rates for lung $(r=0.96)$ cancer in the SFO area. Given that the Program is statewide and assuming that the percentage reduction in cancer incidence also applied statewide, we estimate that the California Tobacco Control Program was associated with about 11,000 less cases of lung cancer statewide in 1989 through 1998. As indicated in Figure 2, this effect grew over time. 


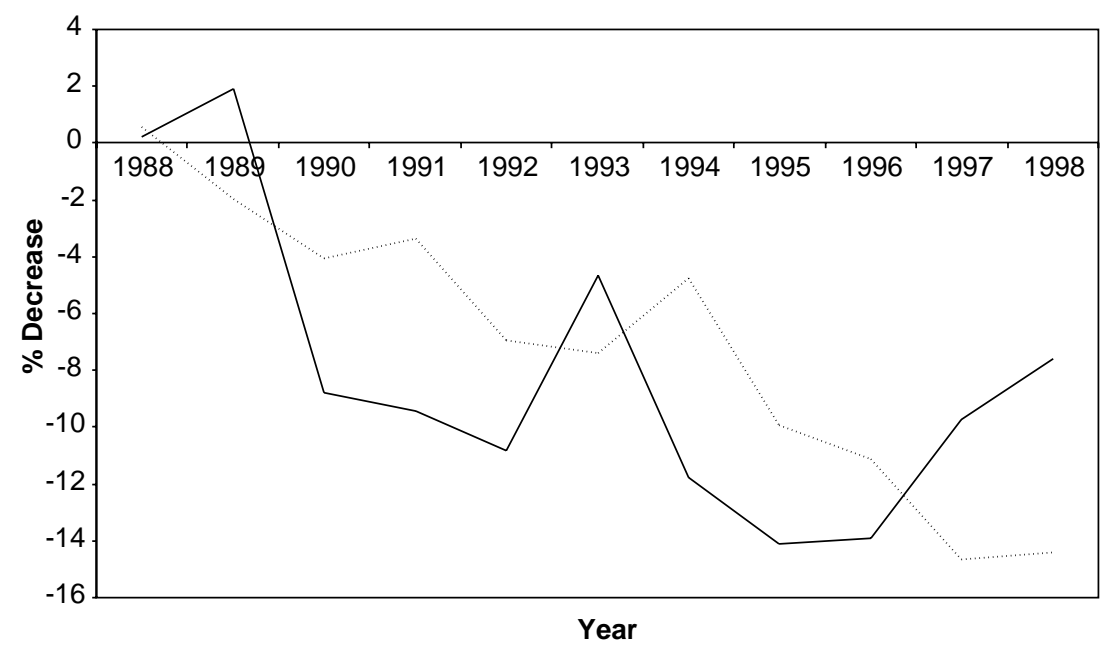

Fig. 2. Percentage reductions in per capita cigarette consumption (solid line) and lung cancer incidence (dotted line) below what would be predicted without the Tobacco Control Program. Changes in cancer incidence are smaller and lag behind changes in cigarette consumption. The relationship is consistent with what would be expected based on individual risk decrease observed with smoking cessation.

\section{Discussion}

In this study, using the SEER database, we found that the California Tobacco Control Program was associated with a significant acceleration in the decline of incidence of lung and probably bladder cancer. Despite the fact that the lung cancer rates in California were already decreasing before the Program was introduced in 1988, the Program came to accelerate this decline, leading to a higher number of cases prevented. Our results are consistent with previous findings of a decline in the incidence of lung cancer in California [10-12], and substantially strengthen the case that the Tobacco Control Program accelerated the decline in cancer incidence because we account for long-term secular trends that were established before the Program was created, changes in underlying patterns of disease incidence and diagnosis, and include "control" groups in two ways. First, that the California Program was not associated with changes in lung cancer incidence in locations outside California. Second, that there was no association with the age-adjusted incidence of two nontobacco caused cancers - prostate and brain - inside California. These results compliment the earlier work that demonstrated that the Program was associated with a decrease in the death rate from heart disease as well as in the smoking prevalence $[3,5,6]$.

One might argue that the changes in cancer incidence we find associated with the Tobacco Control Program begin to appear too soon after the implementation of the program, given the lag between exposure and development of cancer. Eighty to $90 \%$ of lung cancer [13] cases are attributable to smoking. According to the 1990
Surgeon General's Report on the Health Benefits of Smoking Cessation [22] the risk of lung cancer decreases after smoking cessation, falling to $30 \%$ to $50 \%$ of that of a continuing smoker by ten years [22]. Recent studies demonstrate that the risk of lung cancer begins falling relatively soon after smoking cessation. Analysis of the prospective American Cancer Society CPS-I data set shows that excess lung cancer risk begins falling soon after smoking cessation, falling by $10 \%$ in the first two years and by $40 \%$ in five years [23]. Data from the prospective Nurses Health Study [24] found a decrease in lung cancer risk in former smokers compared to current smokers. A $60 \%$ decrease in lung cancer risk was found as soon as two years after quitting, with the decrease in risk increasing over time. Prospective data from the Iowa Women's Health Study agree with these findings [25]. Even in old studies [26] based on purely epidemiological observations in which many people stopped smoking because they were sick (in which the risk of lung cancer increased during the first year following cessation), there was a $22 \%$ drop in risk by year four (compared to years 0 and 1 following cessation). These studies report larger drops in risk than we observed. With a longer period of abstinence there is an increasing decline in risk, which is also reflected in our results (Figure 1). This lag may also explain why the cancer rate is still falling below what was predicted without the Program, even though effects on consumption were dropping off (because of cutbacks to the Program [1]). The change in lung cancer incidence is consistent with what would be expected based on individual risks associated with smoking (Figure 2), 
particularly after considering the fact that there is some lag between the decrease in cigarette consumption and cancer incidence [8].

Bladder cancer incidence rates also fell in California from 1988 to 1999 [12]; rates decreased $12.4 \%$ (from 21.7 to 19 cases per 100,000 persons). As with lung cancer incidence rates reported previously [10-12], one cannot attribute this entire decrease to the Tobacco Control Program because of differences in baseline rates and secular trends between California and the rest of the United States that were established prior to the Program. The marginally significant acceleration in the rate of decline in bladder cancer incidence we found associated with the Program, however, suggests that there was an effect. The smaller effect we found for bladder cancer is reasonable since the risk of bladder cancer associated with smoking is much lower than lung cancer. The relative risk of bladder cancer is only $20-25 \%$ that of lung cancer ( 6 and 25 , respectively [ 13 , $27,28])$.

\section{Biological plausibility}

Our results are biologically plausible. We first found an acceleration of the decrease in lung cancer incidence about two years after the program started. Six months to one year after quitting there is a decrease in biomarkers of lung epithelial cell proliferation. The rate of lung epithelium metaplasia (a pre-cancerous lesion [28]) declines significantly six months after quitting [29]. Cell proliferation markers (Ki-67 BLI, Ki-67 PLI, and metaplasia index) significantly decreased one year after quitting [30]. Consistent with these biological results, the epidemiological data indicates about a $40 \%$ decrease in lung cancer risk five years after quitting compared to current smokers [23-25]. Our second, subsidiary, analysis (Figure 2) that relates the changes in lung cancer incidence with changes in cigarette consumption shows that the magnitude of the changes in lung cancer incidence were consistent with and lagged behind the changes in cigarette consumption on a state level. Adult smoking prevalence decreased in California from 22.8\% in 1988 to $18.0 \%$ in 1999 , a $21 \%$ decline, most of which occurred in the first five years of the program [31]. Therefore, we would expect about a $8 \% \quad(40 \% \times$ $21 \%=8 \%$ ) decrease in the lung cancer incidence over the period we analyzed, which is similar to the $6 \%$ decrease found in our study.

\section{Limitations}

Our study has several limitations. The SEER database is based on data from selected geographic regions, therefore it might not represent incidence rates nationally or statewide. The statistical model we use does not explicitly allow for lags between onset and quitting smoking and changes in cancer incidence. In interpreting our results, it is important to emphasize the fact that we do not conclude that the entire effect of the reduction in lung cancer that we detected appeared in just two years. What we find is that the incidence begins to deviate from what would be expected based on the historical relationship between SFO and the other SEER locations two years after the California Tobacco Control Program was implemented. This finding is consistent with both epidemiological observations on patterns of individual risk following smoking cessation as well as recent biological evidence.

This study seeks to investigate the association between the overall California Tobacco Control Program and cancer incidence. The Program itself included many elements, including a tax increase, mass media campaign, development of smoke-free policies, school programs, and direct cessation. This analysis does not seek to determine the relative importance of these different programmatic elements on cancer incidence. In addition, some of the changes in cancer incidence are probably due to smoking cessation, others due to reduced consumption among continuing smokers, and some due to reduced second-hand smoke exposure. Our analysis does not distinguish between these effects.

This ecologic analysis is based on changes observed in the entire population rather than changes in smoking behavior and second-hand smoke exposure at the individual level [32]. Despite this limitation, our analysis is suitable for detecting an association between the tobacco control program and smoking-induced cancer incidence.

\section{Conclusion}

In conclusion, a comprehensive tobacco prevention and education program is associated with a lower incidence of lung and bronchus cancer and, probably, bladder cancer. The fact that the California Tobacco Control Program was not associated with reductions in the incidence of cancers not caused by tobacco or reductions in lung cancer incidence outside California increases the confidence we can have in the estimate that the Program was associated with 11,000 less cases of lung cancer during its first decade. Funding for comprehensive state tobacco control programs is being cut nationwide [33]. Our results suggest that these cuts will mean more cases of lung cancer as well as more heart disease deaths $[5,6]$. 


\section{References}

1. Glantz SA, Balback E (2000) Tobacco War: Inside the California Battles. Berkeley: University of California.

2. Bal DG, Kizer KW, Felten PG, Mozar HN, Niemeyer D (1990) Reducing tobacco consumption in California. Development of a statewide anti-tobacco use campaign. Jama 264(12): 1570-1574.

3. Pierce JP, Gilpin EA, Emery SL, et al. (1998) Has the California tobacco control program reduced smoking? Jama 280(10): 893899.

4. Lightwood JM, Glantz SA (1997) Short-term economic and health benefits of smoking cessation: myocardial infarction and stroke. Circulation 96(4): 1089-1096.

5. Fichtenberg CM, Glantz SA (2000) Association of the California Tobacco Control Program with declines in cigarette consumption and mortality from heart disease. $N$ Engl J Med 343(24): 17721777.

6. Fichtenberg CM, Glantz SA (2001) Controlling tobacco use. $N$ Engl J Med 344: 1798-1799.

7. American Cancer Society (2001) Cancer Facts \& Figures 2001. Report. Atlanta, GA: ACS.

8. Thun MJ, Henley SJ, Calle EE (2002) Tobacco use and cancer: an epidemiologic perspective for geneticists. Oncogene 21(48): 73077325.

9. Alberg AJ, Samet JM (2003) Epidemiology of Lung Cancer. Chest 123(90010): 21S-S49.

10. Jemal A, Cokkinides VE, Shafey O, Thun MJ (2003) Lung cancer trends in young adults: an early indicator of progress in tobacco control (United States). Cancer Causes Control 14: 579-585.

11. Centers for Disease Control and Prevention (2000) Declines in Lung Cancer Rates - California, 1988-1997. MMWR Weekly Mortality Rep 49(47): 1066-1069.

12. Scott L, Cowling D, Schumacher J, Kwong S, Hoegh H. Tobacco and Cancer in California, 1988-1999 (2003 August) Sacramento, CA: California Department of Health Services, Cancer Surveillance Section.

13. USDHHS. The Health Consequences of Smoking: Cancer. A report of the Surgeon General: U.S. Department of Health and Human Services, Public Health Service, Office on Smoking and Health; 1982. Report No.: DHHS Publication Number (PHS) 8250179 .

14. Levi F, La Vecchia C (2001) Tobacco smoking and prostate cancer: time for an appraisal. Ann Oncol 12(6): 733-738.

15. Lotufo PA, Lee IM, Ajani UA, Hennekens CH, Manson JE (2000) Cigarette smoking and risk of prostate cancer in the physicians' health study (United States). Int J Cancer 87(1): 141-144.

16. Zheng T, Cantor KP, Zhang Y, Chiu BC, Lynch CF (2001) Risk of brain glioma not associated with cigarette smoking or use of other tobacco products in Iowa. Cancer Epidemiol Biomarkers Prev 10(4): 413-414.

17. Ries L, Eisner M, Kosary C, et al. (2002) SEER Cancer Statistics Review, 1973-1999. Bethesda, MD: National Cancer Institute.

18. Orzechowski and Walker, eds. (2001) The Tax Burden on Tobacco. Arlington, Virginia.
19. Eberhardt M, Ingram D, Makuc D, et al. (2001) Urban and Rural Health Chartbook. Health, United States, 2001. Hyattsville, Maryland: National Center for Health Statistics.

20. Center for Health Statistics (1999) Heart disease deaths, California 1997. Sacramento: California: California Department of Health Services. Report No.: DS99-09001.

21. Aguirre-Molina M, Molina CW, Enid Zambrana R, eds. (2001) Health Issues in the Latino Community. San Francisco: JosseyBass.

22. USDHHS. (1990) The Health Benefits of Smoking Cessation: A Report of the Surgeon General, 1990. U.S. Department of Health and Human Services, Public Health Service, Centers for Disease Control, Center for Chronic disease Prevention and Health Promotion, Office on Smoking and Health. Report No.: DHHS Publication No. (CDC) 90-8416.

23. Burns DM (2000) Primary prevention, smoking, and smoking cessation: implications for future trends in lung cancer prevention. Cancer 89(11 Suppl): 2506-2509.

24. Speizer FE, Colditz GA, Hunter DJ, Rosner B, Hennekens C (1999) Prospective study of smoking, antioxidant intake, and lung cancer in middle-aged women (USA). Cancer Causes Control 10(5): 475-482.

25. Ebbert JO, Yang P, Vachon CM, et al. (2003) Lung cancer risk reduction after smoking cessation: observations from a prospective cohort of women. J Clin Oncol 21(5): 921-926.

26. Brown CC, Chu KC (1987) Use of multistage models to infer stage affected by carcinogenic exposure: example of lung cancer and cigarette smoking. J Chronic Dis 40 (Suppl 2): 171S-179S.

27. Rockhill B, Colditz GA (2000) Smoking. In: Colditz GA, ed. Cancer Prevention: The Causes and Prevention of Cancer. The Netherlands: Kluwer Academy Publishers, pp. 3-13.

28. Stratton K, Shetty P, Wallace R, Bondurant S, eds. (2001) Clearing the Smoke. Washington, D.C.: National Academy Press.

29. Lee JS, Lippman SM, Benner SE, et al. (1994) Randomized placebo-controlled trial of isotretinoin in chemoprevention of bronchial squamous metaplasia. J Clin Oncol 12(5): 937-945.

30. Lee JJ, Liu D, Lee JS, et al. (2001) Long-term impact of smoking on lung epithelial proliferation in current and former smokers. JNCI Cancer Spectrum 93(14): 1081-1088.

31. California Department of Health Services. (2002) Fact sheet on adult Smoking prevalence in California. California Department of Health Services. Access Date: March 8, 2003. URL: http:// www.dhs.ca.gov/tobacco/documents/AdultSmoking.pdf.

32. Rothman KJ, Greenland S. (1998) Modern Epidemiology, 2nd edn. Philadelphia: Lippincott Williams \& Wilkins.

33. Campaign for Tobacco Free Kids, American Lung Association, American Cancer Society, American Heart Association. (2002) Show us the money: A Mid-year update on the states' allocation of the tobacco settlement dollars. National Center for Tobacco Free Kids. July 22, 2002. Access Date: August 4. URL: http:// www.tobaccofreekids.org/reports/settlements/2002mid/2002midreport.pdf. 